\title{
INTEGRAÇÃO DE REDES GNSS: UMA PROPOSTA METODOLÓGICA DE DENSIFICAÇÃO DA REDE SIRGAS NA AMÉRICA DO SUL
}

\author{
GNSS network integration: a methodological proposal of the densification of the \\ SIRGAS network in South America \\ MARIA LÍGIA CHUERUBIM \\ JoÃo CARLOS CHAVES \\ JoÃo FranCISCO GALERA MONICO \\ Universidade Estadual Paulista - UNESP \\ Rua Roberto Simonsen, 305. Caixa Postal 467 \\ CEP - 19060-900 - Presidente Prudente - SP \\ mchuerubim@gmail.com; \{jcchaves, galera\}@fct.unesp.br
}

\begin{abstract}
RESUMO
A característica continental do Brasil implica na necessidade de constantes desafios científicos em pesquisas espaciais e tecnológicas, que visam contribuir ao engajamento de centros de pesquisas, fornecendo instrumentos às atividades espaciais no país e no mundo, ampliando as perspectivas de aplicação de técnicas geodésicas como o GPS (Global Positioning System). Neste contexto, destaca-se a contribuição ao IGS (International GNSS Service) dos centros de locais de análise SIRGAS (Sistema de Referência Geocêntrico para as Américas) e o IGS-RNAACSIR (IGS Regional Network Associate Analysis Centre for SIRGAS), que disponibilizam soluções semanais no formato SINEX (Software INdependent EXchange Format), referenciadas ao ITRS (International Terrestrial Reference System), que posteriormente são integradas no contexto de uma solução regional pelo DGFI (Deutsches Geodätiches Forschungsinstitut), denominada IGS-RNAACSIR. Nesta perspectiva, este projeto apresenta uma metodologia de integração de redes GNSS locais no contexto de uma solução regional para o Brasil, com base em dados gerados pelas redes ativas RBMC (Rede Brasileira de Monitoramento Contínuo), GNSS SP (Rede GPS Ativa do Oeste do Estado de São Paulo, e em soluções SIRGAS no formato SINEX, utilizando o princípio da combinação das equações normais pelo MMQ (Método dos Mínimos Quadrados), contribuindo com a manutenção e densificação da rede SIRGAS na América Latina.
\end{abstract}


Palavras-chave: Integração de Redes GNSS; Combinação de Equações Normais; SIRGAS; SINEX.

\begin{abstract}
The continental characteristic of Brazil implies in the necessity of constant scientific challenges in space and technological research, contributing to the development and enrollment of national and international centers of research, supplying instruments to the space activities in the country and extending the perspectives of application of geodetic techniques as GPS (Global Positioning System). In this context, it is remarkable the contribution of the local analysis centers of SIRGAS (System of Geocentric Reference for Americas) and the IGS-RNAAC-SIR (Regional IGS Network Associate Analysis Centre for SIRGAS) with IGS (International GNSS Service), that compute weekly solutions in SINEX format (Software Independent Exchange Format), referenced to ITRS (International Terrestrial Reference System), that are later integrated in the context of a global solution for IGS. In this perspective, this paper presents a methodology of the integration of local GNSS networks in the context of a regional solution for Brazil, based on active GNSS networks, likes RBMC (Brazilian Network of Continuous Monitoring) and GNSS SP (Active Network GPS of the west of the State of São Paulo), and in SIRGAS solutions in SINEX format, using the principle of the combination of the normal equations for the least squares adjustment (LSQ), contributing to the maintenance and densification of SIRGAS-CON network in Latin America.
\end{abstract}

Keywords: Integration of GNSS Networks; Combination of Normal Equations; SIRGAS; SINEX.

\title{
1. INTRODUÇÃO
}

Explorar a potencialidade das técnicas geodésicas espaciais como Global Navigation Satellite System (GNSS); Satellite Laser Ranging (SLR), Lunar Laser Ranging (LLR), Very Long Baseline Interferometry (VLBI), Doppler Orbitography and Radio Positioning Integrated by Satellite (DORIS) para a investigação de variações globais ou regionais, fenômenos sazonais ou seculares, possibilita que estes referenciais sejam realizados com uma alta acurácia. Assim, Centros de Análise e Processamento devem utilizar padrões adotados internacionalmente como: correção absoluta das variações do centro de fase da antena; modelagem do atraso zenital troposférico com base em funções de mapeamento; correções dos efeitos de $2^{\mathrm{a}}$ e $3^{\mathrm{a}}$ ordem da ionosfera; influências das cargas oceânicas, entre outros (STEINGENBERGER et al., 2006).

No que se refere a um sistema regional, o Sistema de Referência Geocêntrico para as Américas (SIRGAS) vem disponibilizando soluções semanais no formato SINEX, referenciadas ao ITRS, das estações da rede SIRGAS-CON (Rede de Operação Contínua SIRGAS), densificação da rede ITRF na América Latina e região do Caribe, com base nos processamentos dos centros locais, como o IBGE 
(Instituto Brasileiro de Geografia e Estatística), o Instituto Geográfico Agustin Codazzi (IGAC, Colômbia) e o Centro de Procesamiento Ingeniería-MendozaArgentina de la Universidad Nacional del Cuyo (CIMA, Argentina), que caracterizam as redes de densificação SIRGAS (SIRGAS-CON-D), respectivamente, na América Central, parte da América do Norte América do Sul e região do Caribe. Essas soluções são, posteriormente, combinadas pelo DGFI (Deutsches Geodätiches Forschungsinstitut), Centro de Análise Regional IGS (International GNSS Service) denominado IGS- RNAAC- SIR (Regional Network Associate Analysis Center for SIRGAS), responsável por processar a rede regional SIRGAS-CON-C (Rede de Operação Contínua SIRGAS), (SIRGAS, 2009).

Este trabalho tem por objetivo propor uma metodologia de integração de redes locais no contexto de uma solução regional para o Brasil, que forneça a manutenção e densificação do SIRGAS na América do Sul, com base nos processamentos semanais das estações GPS pertencentes às redes RBMC e Rede GNSS SP (Rede GPS Ativa do oeste do Estado de São Paulo), com o intuito de monitorar a variação temporal das coordenadas destas estações, bem como a qualidade dos dados disponibilizados para a densificação desta rede.

\section{FUNDAMENTOS E CONCEITOS ENVOLVIDOS NO TRABALHO}

Os fundamentos básicos necessários ao desenvolvimento desta pesquisa são apresentados, dentre eles os conceitos e modelos matemáticos em que se fundamenta a metodologia proposta neste trabalho.

\subsection{Integração de Redes}

Neste trabalho, o termo integração de redes refere-se à densificação de uma rede geodésica regional, como por exemplo, a rede SIRGAS-CON a partir do estabelecimento de redes geodésicas locais, caso da RBMC e da Rede GNSS SP. Para tanto, os vértices com coordenadas conhecidas no referencial a ser densificado (estações de conexão), farão parte da rede a ser integrada, e serão introduzidos como injunções no ajustamento. Desta forma, será apresentado o ajustamento das redes geodésicas GNSS utilizando o conceito de ajustamento pelo Método dos Mínimos Quadrados (MMQ) para sistemas não lineares, aplicando o método paramétrico com a inclusão de novas observações e parâmetros (GEMAEL, 1994), com base na combinação das equações normais.

\subsection{Modelos matemáticos envolvidos no processo de integração de redes geodésicas}

No processamento e ajuste de redes geodésicas de alta precisão, o método de posicionamento normalmente empregado é o relativo estático, utilizando as equações de observação de fase e/ou pseudodistância. No processamento, são aplicados os modelos das Duplas - Diferenças (DDs) das observáveis GPS para corrigir erros comuns às estações. Desta forma, considerando-se uma rede geodésica 
constituída por " $r$ " receptores e considerando-se " $s$ " satélites rastreados em " $n$ " épocas distintas, tem-se que o vetor das observações formados entre as DDs de fase, tomando o satélite 1 como satélite base, tem-se que o vetor do conjunto de observações será dado por:

$$
\begin{gathered}
L_{b}=\left[\Delta \phi_{1,2}^{1,2}\left(t_{1}\right), \Delta \phi_{1,2}^{1,3}\left(t_{1}\right), \ldots, \Delta \phi_{1,2}^{1, s}\left(t_{1}\right), \ldots,\right. \\
\Delta \phi_{1, r}^{1,2}\left(t_{1}\right), \Delta \phi_{1, r}^{1,3}\left(t_{1}\right), \ldots, \Delta \phi_{1, r}^{1, s}\left(t_{1}\right), \Delta \phi_{1,2}^{1,2}\left(t_{2}\right), \\
\Delta \phi_{1,2}^{1,3}\left(t_{2}\right), \ldots, \Delta \phi_{1,2}^{1, s}\left(t_{2}\right), \ldots, \Delta \phi_{1, r}^{1,2}\left(t_{2}\right), \Delta \phi_{1, r}^{1,3}\left(t_{2}\right), \ldots, \\
\Delta \phi_{1, r}^{1, s}\left(t_{2}\right), \ldots, \Delta \phi_{1,2}^{1,2}\left(t_{n}\right), \Delta \phi_{1,2}^{1,3}\left(t_{n}\right), \ldots, \\
\left.\Delta \phi_{1,2}^{1, s}\left(t_{n}\right), \ldots, \Delta \phi_{1, r}^{1,2}\left(t_{n}\right), \Delta \phi_{1, r}^{1,3}\left(t_{n}\right), \ldots, \Delta \phi_{1, r}^{1, s}\left(t_{n}\right)\right]
\end{gathered}
$$

onde $\Delta \phi_{1, r}^{1, s}(t)=\phi_{1, r}^{s}(t)-\phi_{1, r}^{1}(t)$. Nesta perspectiva, a Matriz Variância-Covariância (MVC) do conjunto de observações será definida pela precisão de cada observação $\left(\sigma^{2}\left(\phi_{1, r}^{1, s}(t)\right)\right)$ e pela correlação existente entre esse conjunto de observações $\left(\sigma^{2}\left(\phi_{1, r}^{1, s}(t), \phi_{1, r}^{1, s-1}(t)\right)\right)$ em uma determinada época. Portanto, a MVC das DDs com base no conjunto de observações (Equação 1), considerando-se, como forma de exemplificação, a formação de linhas de base independentes para uma rede constituída por 3 receptores rastreando simultaneamente 4 satélites em 3 épocas distintas, será dada por:

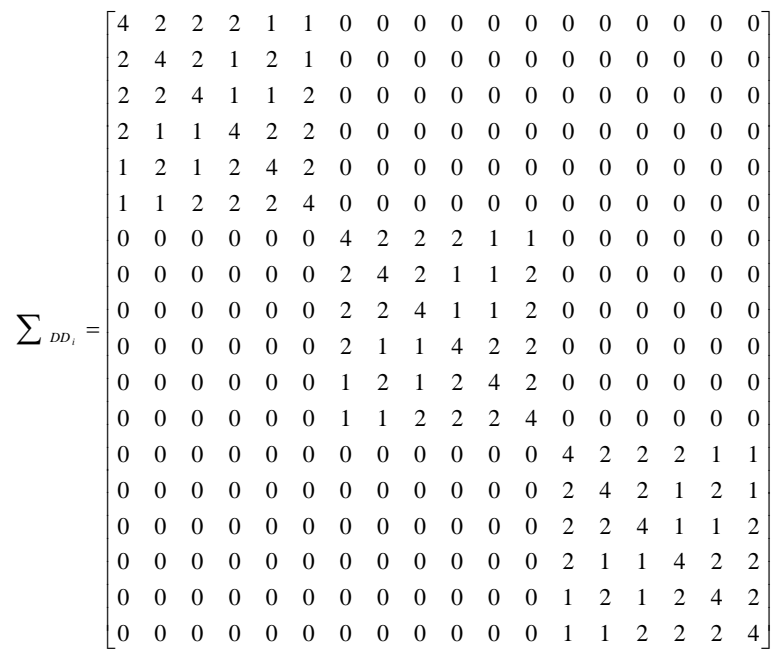

Bol. Ciênc. Geod., sec. Artigos, Curitiba, v. 17, nº 4, p.477-495, out-dez, 2011. 
Desta forma obtém-se a matriz Peso (Equação 3) do conjunto de observações:

$$
P=\sigma_{0}^{2} \sum_{D D_{i}}^{-1}
$$

O vetor dos parâmetros incógnitos será dado por:

$$
\begin{gathered}
X_{a}=\left[\Delta X_{1}, \Delta Y_{1}, \Delta Z_{1}, \Delta X_{2}, \Delta Y_{2}, \Delta Z_{2}, \ldots, \Delta X_{r}, \Delta Y_{r}, \Delta Z_{r},\right. \\
\left.\Delta N_{1,2}^{1,2}, \Delta N_{1,2}^{1,3}, \ldots, \Delta N_{1,2}^{1, s}, \ldots, \Delta N_{1, r}^{1,2}, \Delta N_{1, r}^{1,3}, \ldots, \Delta N_{1, r}^{1, s}\right]
\end{gathered}
$$

onde $\Delta X_{r}=X_{r}-X_{r^{0}}$. A matriz design $A$ (matriz Jacobiana), será dada pelas derivadas parciais em relação às componentes $X, Y$ e $Z$ de cada estação. Com base na matriz $A$, na matriz Peso (Equação 3) e no vetor de observações (Equação $1)$, obtêm-se as equações normais $N_{L}=(u \times u)$ e $U_{L}=(u \times 1)$ da rede local (GEMAEL, 1994).

Posteriormente, para realizar a integração dessas soluções com uma solução regional torna-se necessária a reconstrução das equações normais a partir das informações contidas nos arquivos SINEX da rede regional, como o fator de variância a posteriori $\left(\hat{\sigma}_{0}^{2}\right)$, parâmetros ajustados $\left(X_{a}^{R}\right)$ e aproximados $\left(X_{0}^{R}\right), \operatorname{MVC}$ dos parâmetros ajustados $\left(\sum X_{a}\right)$, bem como de um número mínimo de estações comuns a ambas as redes. Desta forma, com base na $\sum X_{a}$ e no $\hat{\sigma}_{0}^{2}$, obtém-se a $N_{R}$ da rede regional:

$$
\sum X_{a}=\hat{\sigma}_{0}^{2} N_{R}^{-1} \Rightarrow N_{R}=\hat{\sigma}_{0}^{2} \sum X_{a}^{-1}
$$

E, a partir dos conjuntos de $X_{a}^{R}$ e $X_{0}^{R}$, obtém-se o vetor $X_{R}$ :

$$
X_{a}^{R}=X_{0}^{R}+X_{R} \Rightarrow X_{R}=X_{a}^{R}-X_{0}^{R},
$$

com base no qual se torna possível obter a matriz $U_{R}$ pela seguinte Equação:

$$
X_{R}=-N_{R}^{-1} U_{R}
$$

Multiplicando-se ambos os lados da Equação 7 por $N_{R}$ tem-se: 


$$
N_{R} X_{R}=-N_{R}^{-1} N_{R} U_{R} \Rightarrow U_{R}=-N_{R} X_{R} \text {. }
$$

Com essas informações, a integração entre redes regionais e locais deve ser realizada em termos de suas equações normais obtidas, respectivamente, do processamento dos dados GPS e dos arquivos SINEX. Para tanto, tais soluções devem estar livres de injunções ou de fracamente injuncionadas, caso contrário, uma solução de forte injunção poderá deformar a geometria da rede e, consequentemente, comprometer a integração entre as mesmas. No processo de combinação de redes, em geral, o número de estações envolvidas varia a cada época, o que conduz a necessidade de se expandir o sistema de equações normais, alocando-se zeros nas linhas e colunas correspondentes aos respectivos parâmetros.

Portanto, com base em uma série temporal obtida a partir de várias soluções semanais, em uma determinada época de referência $\left(t_{0}\right)$, podem-se estimar as coordenadas de um conjunto de estações e suas respectivas velocidades, em uma determinada época t, com base em um conjunto de vetores-posição $X_{t_{1}}, X_{t_{2}}, \ldots, X_{t_{n}}$ e suas respectivas MVCs,

$\left(\sum X_{i=t_{1} . t_{2}, \ldots, t_{n}}\right)$, a partir de $n$ observações, ou seja:

$$
\begin{gathered}
X_{t_{1}}=X_{t}+V_{t}\left(t-t_{1}\right) \\
Y_{t_{1}}=Y_{t}+V_{t}\left(t-t_{1}\right) \\
Z_{t_{1}}=Z_{t}+V_{t}\left(t-t_{1}\right) \\
\vdots \\
X_{t_{n}}=X_{t}+V_{t}\left(t-t_{n}\right) \\
Y_{t_{n}}=Y_{t}+V_{t}\left(t-t_{n}\right) \\
Z_{t_{n}}=Z_{t}+V_{t}\left(t-t_{n}\right)
\end{gathered}
$$

em que cada $\vec{X}_{t_{n}}$ corresponde à solução semanal integrada na época $t$. Logo, considerando-se $K$ épocas e $n$ estações, tem-se a seguinte representação matricial: 
$\left[\begin{array}{c}\vec{V}_{X_{1}^{1}} \\ \vec{V}_{X_{1}^{2}} \\ \vdots \\ \vec{V}_{X_{1}^{k}} \\ \vdots \\ \vec{V}_{X_{n}^{1}} \\ \vec{V}_{X_{n}^{2}} \\ \vdots \\ \vec{V}_{X_{n}^{k}}\end{array}\right]=\left[\begin{array}{ccccc}I & \Delta t^{1} I & \ldots & 0 & 0 \\ I & \Delta t^{2} I & \ldots & 0 & 0 \\ \vdots & \vdots & \ldots & 0 & 0 \\ I & \Delta t^{k} I & \ldots & 0 & 0 \\ \vdots & \vdots & \ldots & \vdots & \vdots \\ 0 & 0 & \ldots & I & \Delta t^{1} I \\ 0 & 0 & \ldots & I & \Delta t^{2} I \\ 0 & 0 & \ldots & \vdots & \vdots \\ 0 & 0 & \ldots & I & \Delta t^{k} I\end{array}\right]\left[\begin{array}{l}\vec{X}_{1}^{0}\left(t_{0}\right) \\ \vec{V}_{1}^{0}\left(t_{0}\right) \\ \vdots \\ \vec{X}_{n}^{0}\left(t_{0}\right) \\ \vec{V}_{n}^{0}\left(t_{0}\right)\end{array}\right]$

$$
-\left[\begin{array}{c}
\vec{X}_{i}\left(t_{1}\right)-\vec{X}_{i}^{0}\left(t_{0}\right)-\vec{V}_{X_{i}}^{0} \Delta t^{1} \\
\vec{X}_{i}\left(t_{2}\right)-\vec{X}_{i}^{0}\left(t_{0}\right)-\vec{V}_{X_{i}}^{0} \Delta t^{2} \\
\vdots \\
\vec{X}_{i}\left(t_{k}\right)-\vec{X}_{i}^{0}\left(t_{0}\right)-\vec{V}_{X_{i}}^{0} \Delta t^{k} \\
\vdots \\
\vec{X}_{n}\left(t_{1}\right)-\vec{X}_{n}^{0}\left(t_{0}\right)-\vec{V}_{X_{n}}^{0} \Delta t^{1} \\
\vec{X}_{n}\left(t_{2}\right)-\vec{X}_{n}^{0}\left(t_{0}\right)-\vec{V}_{X_{n}}^{0} \Delta t^{2} \\
\vdots \\
\vec{X}_{n}\left(t_{k}\right)-\vec{X}_{n}^{0}\left(t_{0}\right)-\vec{V}_{X_{n}}^{0} \Delta t^{k}
\end{array}\right]
$$

com $\quad \vec{V}_{X_{t}}=\left[V_{X}(t), V_{Y}(t), V_{Z}(t),\right]^{T}, \quad \vec{X}_{\mathbf{t}}=\left[X_{X}(t), X_{Y}(t), X_{Z}(t)\right]^{T}, \quad$ na $\quad$ época $\quad(t), \quad$ e $\vec{V}_{X_{i}}^{0}=\left[V_{X}\left(t_{0}\right), V_{Y}\left(t_{0}\right), V_{Z}\left(t_{0}\right),\right]^{T}$ e $\vec{X}_{t_{0}}^{0}=\left[X_{X}\left(t_{0}\right), X_{Y}\left(t_{0}\right), X_{Z}\left(t_{0}\right)\right]^{T}$ na época $\left(t_{0}\right)$.

O desenvolvimento das equações de pseudo-observação (Equação 10) baseiase na combinação de soluções semanais GPS, o que conduz à deficiência em orientação solucionada pelo conceito de uma rede sem rotação (NNR - No Net Rotation) (MONICO, 2005). Contudo, o desenvolvimento desta pesquisa compreende estações localizadas em sua maioria na placa litosférica sul americana, o que implica que a aplicação da condição da condição NNR não influenciará nos resultados, uma vez que deve ser aplicada quando se tem um número de estações que representem a Terra em sua globalidade. Aplicou-se, portanto, neste trabalho, a condição de origem (NNT - No Net Translation), por meio dos vértices das estações que estabelecem o datum da rede (MONICO, 2005): 


$$
\begin{aligned}
& \sum_{i=1}^{n} \Delta \vec{X}_{i}=0, \\
& \sum_{i=1}^{n} \Delta \vec{V}_{i}=0
\end{aligned}
$$

onde $\Delta \vec{X}_{i}$ e $\Delta \vec{V}_{i}$ correspondem aos vetores das correções aos parâmetros aproximados e $n$ ao número de estações que estabelecem o datum. Matricialmente essa condição é representada por:

$$
C=\left[\begin{array}{lll}
1 & 0 & 0 \\
0 & 1 & 0 \\
0 & 0 & 1
\end{array}\right],
$$

onde a matriz $C$ apresenta dimensões $3 \times 3$, para cada estação envolvida nessa condição. Verifica-se, que a escolha das estações deve estar atrelada ao seu comportamento geofísico, localização geográfica e período de observação. A condição NNT (Equação 12) é introduzida somente na solução final, nas colunas correspondentes as coordenadas das estações que fazem parte da condição NNT, para que desta forma a solução não seja de forte injunção.

Desta forma, ao aplicar o conceito de NNT (Equação 12) a Equação (10) passará a ser representada por:

$$
\left[\begin{array}{c}
\vec{V}_{X_{1}^{1}} \\
\vec{V}_{X_{1}^{2}} \\
\vdots \\
\vec{V}_{X_{1}^{k}} \\
\vdots \\
\vec{V}_{X_{n}^{1}} \\
\vec{V}_{X_{n}^{2}} \\
\cdots \\
\vec{V}_{X_{n}^{k}} \\
0
\end{array}\right]=\left[\begin{array}{ccccc}
I & \Delta t^{1} I & \cdots & 0 & 0 \\
I & \Delta t^{2} I & \cdots & 0 & 0 \\
\vdots & \vdots & \cdots & 0 & 0 \\
I & \Delta t^{k} I & \cdots & 0 & 0 \\
\vdots & \vdots & \cdots & \vdots & \vdots \\
0 & 0 & \cdots & I & \Delta t^{1} I \\
0 & 0 & \cdots & I & \Delta t^{2} I \\
0 & 0 & \cdots & \vdots & \vdots \\
0 & 0 & \cdots & I & \Delta t^{k} I \\
C_{N N T} & 0 & \cdots & 0 & 0
\end{array}\right]\left[\begin{array}{c}
\vec{X}_{i}^{0}\left(t_{0}\right) \\
\vec{V}_{X_{i}}^{0}\left(t_{0}\right) \\
\vdots \\
\vec{X}_{n}^{0}\left(t_{0}\right) \\
\vec{V}_{X_{n}}^{0}\left(t_{0}\right)
\end{array}\right]-\left[\begin{array}{c}
\vec{X}_{i}\left(t_{1}\right)-\vec{X}_{i}\left(t_{0}\right)-\vec{V}_{X_{i}}^{0} \Delta t^{1} \\
\vec{X}_{i}\left(t_{2}\right)-\vec{X}_{i}\left(t_{0}\right)-\vec{V}_{X_{i}}^{0} \Delta t^{2} \\
\vdots \\
\vec{X}_{i}\left(t_{k}\right)-\vec{X}_{i}\left(t_{0}\right)-\vec{V}_{X_{i}}^{0} \Delta t^{k} \\
\vdots \\
\vec{X}_{n}\left(t_{1}\right)-\vec{X}_{n}\left(t_{0}\right)-\vec{V}_{X_{n}}^{0} \Delta t^{1} \\
\vec{X}_{n}\left(t_{2}\right)-\vec{X}_{n}\left(t_{0}\right)-\vec{V}_{X_{n}}^{0} \Delta t^{2} \\
\vdots \\
\vec{X}_{n}\left(t_{k}\right)-\vec{X}_{n}\left(t_{0}\right)-\vec{V}_{X_{n}}^{0} \Delta t^{k} \\
0
\end{array}\right]
$$

e, por conseguinte, o sistema de equações normais para cada solução individual, será dado por (MONICO, 2006): 


$$
N=A^{T} N_{s} A+C^{T} C=\left[\begin{array}{cc}
\sum_{i=1}^{k} N_{s}^{i}+C^{T} C & \sum_{i=1}^{k} N_{s}^{i} \Delta t_{s}^{i} \\
\sum_{i=1}^{k} N_{s}^{i} \Delta t_{s}^{i} & \sum_{i=1}^{k} N_{s}^{i}\left(\Delta t_{s}^{i}\right)^{2}
\end{array}\right],
$$

onde a matriz peso é igual à equação normal de cada solução semanal, ou seja, $P e s o=N_{\text {sem }}$, em que $k$ representa o número de épocas. Nesta etapa, cada solução individual é conservada livre de injunções, para que possa ser utilizada na obtenção da solução final. Além disso, no processo de combinação de soluções, os valores aproximados de todos os parâmetros envolvidos devem ser os mesmos.

Portanto, o sistema de equações normais para o sistema de Equações (14) será dado por:

$$
\left.\begin{array}{c}
{\left[\begin{array}{ccccc}
\sum_{i=1}^{k} N_{X_{i}}+C^{T} C & \sum_{i=1}^{k} N_{X_{i}} \Delta t_{i} & \ldots & N_{X_{n}, X_{1}}^{i=1, \ldots, k} & N_{X_{n}, X_{1}}^{i=1, \ldots, k} \Delta t_{i} \\
\sum_{i=1}^{k} N_{X_{i}} \Delta t_{i} & \sum_{i=1}^{k} N_{X_{i}}\left(\Delta t_{i}\right)^{2} & \ldots & N_{X_{n}, X_{1}}^{i=1, \ldots, k} \Delta t_{i} & N_{X_{n}, X_{1}}^{i=1, \ldots, k} \\
\vdots & & \ddots & \vdots & \\
N_{X_{n}, X_{1}}^{i=1, \ldots, k} & N_{X_{n}, X_{1}}^{i=1, \ldots, k} \Delta t_{i} & \ldots & \sum_{i=1}^{k} N_{X_{n}} & \sum_{i=1}^{k} N_{X_{n}} \Delta t_{i} \\
N_{X_{n}, X_{1}}^{i=1, \ldots, k} \Delta t_{i} & N_{X_{n}, X_{1}}^{i=1, \ldots, k} & \ldots & \sum_{i=1}^{k} N_{X_{n}} \Delta t_{i} & \sum_{i=1}^{k} N_{X_{n}}\left(\Delta t_{i}\right)^{2}
\end{array}\right]} \\
=\left[\begin{array}{c}
\sum_{i=1}^{k} N_{X_{i}}^{i}\left(\vec{X}_{i}\left(t_{1}\right)-\vec{X}_{i}^{0}\left(t_{0}\right)-\bar{V}_{X_{i}}^{0} \Delta t^{1}\right) \\
\vec{X}_{1}^{0}\left(t_{0}\right) \\
\vec{V}_{1}^{0}\left(t_{0}\right) \\
\vec{X}_{n}^{0}\left(t_{0}\right) \\
\vec{V}_{n}^{0}\left(t_{0}\right)
\end{array}\right]= \\
N_{X_{i}}^{i} \Delta t^{i}\left(\vec{X}_{i}\left(t_{1}\right)-\vec{X}_{i}^{0}\left(t_{0}\right)-\bar{V}_{X_{i}}^{0} \Delta t^{1}\right) \\
\vdots \\
\sum_{i=1}^{k} N_{X_{n}}^{i}\left(\vec{X}_{n}\left(t_{1}\right)-\vec{X}_{n}^{0}\left(t_{0}\right)-\bar{V}_{X_{n}}^{0} \Delta t^{1}\right) \\
\sum_{i=1}^{k} N_{X_{n}}^{i} \Delta t^{i}\left(\vec{X}_{n}\left(t_{1}\right)-\vec{X}_{n}^{0}\left(t_{0}\right)-\bar{V}_{X_{n}}^{0} \Delta t^{1}\right)
\end{array}\right]
$$

Desta forma, os parâmetros que não são comuns às soluções são preenchidos com zero, ou seja, ocorre a expansão para a matriz normal, de tal forma que cada solução semanal contenha os mesmos parâmetros. Assim, ao combinar $n$ soluções semanais (coordenadas) obtêm-se os parâmetros $X_{t}$ e $V_{t}$ de cada estação na época de referência $t$ :

$$
X_{a}=\left[X_{1}, Y_{1}, Z_{1}, V_{X_{1}}, V_{Y_{1}}, V_{Z_{1}}, \ldots, X_{n}, Y_{n}, Z_{n}, V_{X_{n}}, V_{Y_{n}}, V_{Z_{n}}\right]^{T}
$$


Os parâmetros ajustados (Equação 16) compreendem a solução final do ajustamento (coordenadas e velocidades e respectivas precisões) de cada uma das estações com base nas soluções semanais estimadas a cada época.

\section{METODOLOGIA}

Para a estimativa das coordenadas e velocidades das estações, buscou-se corrigir os principais erros que influenciam o posicionamento por satélites como: os efeitos de $1^{\mathrm{a}}$ e $2^{\mathrm{a}}$ ordem da ionosfera; a modelagem do atraso troposférico, com a aplicação de modelos empíricos (Saastamoinen) e funções de mapeamento, tais como a VMF (Viena Mapping Function); a modelagem absoluta das variações do centro de fase das antenas GPS e a aplicação das correções inerentes ao efeito das cargas dos oceanos (FES2004) e da atmosfera. Foram utilizados, para o período de dados (2006 a 2008), os arquivos de observação GPS no formato RINEX das estações Brasília (BRAZ), Bom Jesus (BOMJ), Cuiabá (CUIB), Imperatriz (IMPZ), Manaus (NAUS), Presidente Prudente (PPTE) e Viçosa (VICO) da RBMC, disponibilizados pelo IBGE, no seguinte endereço: ftp://geoftp.ibge.gov.br/ RBMC/dados, no formato RINEX. A escolha dessas estações deve-se ao fato das mesmas terem participado da densificação da rede ITRS, na realização ITRF2000 e, atualmente, por contribuírem à densificação e manutenção da rede SIRGAS na América do Sul. Foram utilizadas, ainda, soluções semanais SIRGAS compreendendo as estações SIRGAS-CON disponíveis para este período, precisamente 193 estações, fracamente injuncionadas (SIRwwww7.SNX), no formato SINEX (ftp://ftp.dgfi.badw-muenchen.de/pub/gps/SIR/), bem como dados das estações Ilha Solteira (ILHA), Ourinhos (OURI), Rosana (ROSA), São José do Rio Preto (SJRP) e Lins (LINS), pertencentes à Rede GNSS SP (http://gege.prudente.unesp.br).

Nesta metodologia, os dados GPS das redes locais no formato RINEX são corrigidos dos efeitos de $2^{\mathrm{a}}$ ordem da ionosfera com o aplicativo RINEX_HO (MARQUES, 2008) e, posteriormente, processados diariamente com o software Bernese 5.0, com base no ajustamento de "rede livre”, sem a definição do datum. Essas soluções diárias são armazenadas e combinadas semanalmente com o módulo ADDNEQ2 do Bernese, que permite a combinação de soluções com base nas equações normais, resultando em soluções semanais locais livres de injunção (BERNESE, 2007). As soluções semanais da rede local, posteriormente, podem ser integradas a uma rede de âmbito regional como a rede SIRGAS-CON. Para tanto, basta haver estações comuns entre as mesmas e utilizar soluções disponibilizadas pela rede regional em formatos que possibilitem a aplicação desta metodologia, como o formato SINEX.

Neste trabalho, foram utilizadas as soluções semanais SIRGAS fracamente injuncionadas (loosely constrained), cujas equações normais foram reconstruídas utilizando-se o módulo SNX2NQ0 do Bernese (BERNESE, 2007). A integração entre essas redes foi feita com o módulo ADDNEQ2 de duas formas: rede livre (free 
network) e coordenadas injuncionadas (coordinates constrained). O primeiro caso, aplica-se às soluções multi-anuais, em que se obtém coordenadas e velocidades das estações para um período maior e igual a três anos de dados. As soluções injuncionadas, destinam-se a fins práticos como atividades que envolvam posicionamento. Nessas soluções, as coordenadas das estações que definem o datum são injuncionadas e estimadas juntamente com as coordenadas das estações envolvidas no processamento.

\section{EXPERIMENTOS E RESULTADOS}

Nesta Seção, são apresentados os experimentos e os resultados obtidos na pesquisa. O primeiro conjunto de experimentos refere-se à correção e análise do impacto do efeito de $2^{a}$ ordem da ionosfera, para o período de 2006 a 2008, na estimativa das coordenadas das estações. Em seguida, são apresentadas as soluções obtidas (coordenadas e velocidades) com base na metodologia proposta neste trabalho.

\subsection{Correção do efeito de $2^{a}$ da ionosfera}

A aplicação da correção do efeito de $2^{a}$ ordem da ionosfera foi realizada com base na no software RINEX_HO, que permite a correção deste efeito nas observáveis GPS, com base no TEC calculado a partir da pseudodistância, o qual é interpolado a partir dos GIMs, disponibilizados via Internet (http://www.aiub.unibe.ch/download/CODE/), e nas informações associadas ao campo geomagnético da Terra. Verificou-se, com base nos resultados obtidos, que a magnitude obtida para os efeito de $2^{\mathrm{a}}$ ordem nas observáveis GPS é em média de 4 $\mathrm{mm}$.

Posteriormente, analisou-se a magnitude deste efeito no posicionamento relativo, comparando as discrepâncias entre os resultados obtidos utilizando os arquivos RINEX sem a correção do efeito de $2^{a}$ ordem e os arquivos corrigidos com o RINEX_HO, como se observa nas séries temporais da estação PPTE ilustradas nas Figuras 1 a 3:

Com base neste experimento verificou-se que a discrepância entre essas soluções é milimétrica para o período de 2006 a 2008, atingindo valores maiores para a componente altimétrica variando de varia de 1 a $4 \mathrm{~mm}$, valores estes coerentes com os encontrados nos experimentos na literatura (FRITSCHE et al., 2005). 
Figura 1 - Discrepância na componente Leste entre as soluções obtidas com e sem correção dos efeitos de $2^{\mathrm{a}}$ e $3^{\mathrm{a}}$ ordem da ionosfera para a estação PPTE.

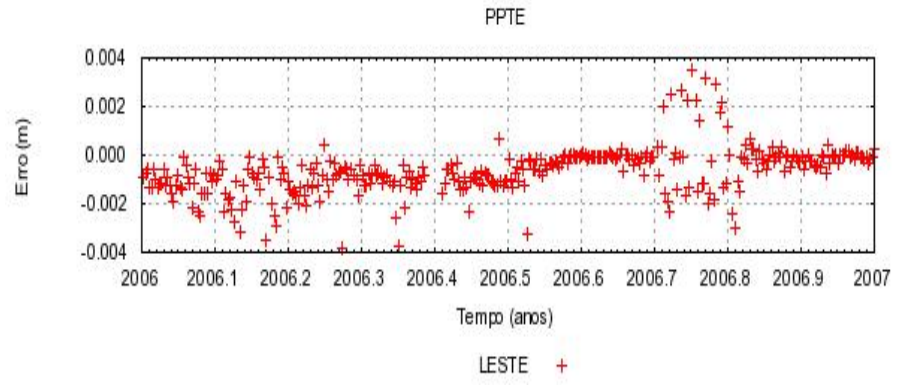

Figura 2 - Discrepância na componente Norte entre as soluções obtidas com e sem correção dos efeitos de $2^{\mathrm{a}}$ e $3^{\mathrm{a}}$ ordem da ionosfera para a estação PPTE.

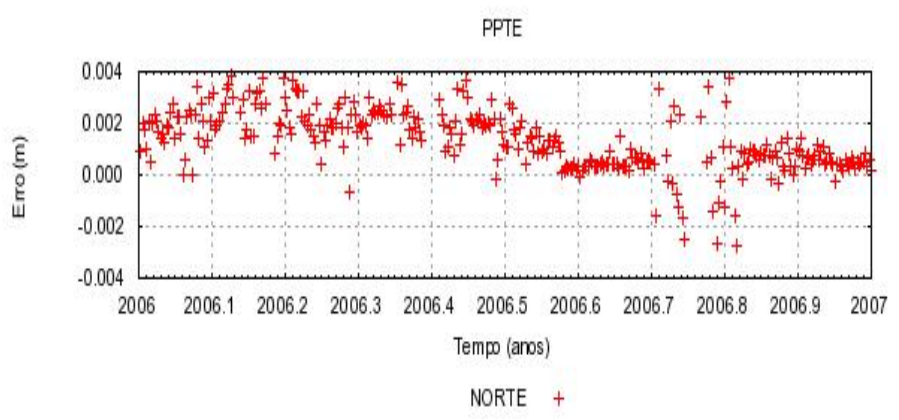

Figura 3 - Discrepância na componente Vertical entre as soluções obtidas com e sem correção dos efeitos de $2^{\mathrm{a}} \mathrm{e} 3^{\mathrm{a}}$ ordem da ionosfera para a estação PPTE.

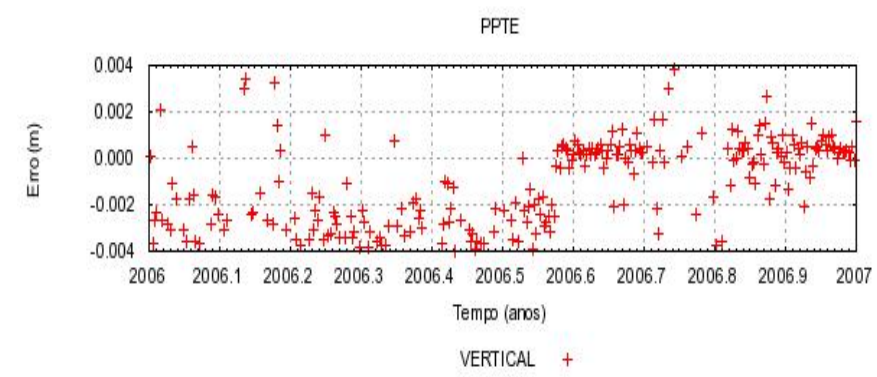

Bol. Ciênc. Geod., sec. Artigos, Curitiba, v. 17, nº 4, p.477-495, out-dez, 2011. 


\subsection{Obtenção das soluções integradas}

Nesta Seção são apresentados os resultados obtidos com o software Bernese, com base na metodologia de integração de redes apresentada na Seção 3, resultando na estimativa das coordenadas de 193 estações, obtidas a partir do processamento de 2006 a 2008, na época 2007,507. Dessas estações apenas 92 tiveram suas velocidades estimadas com base em critérios como: período de atividade e quantidade de dados maior e igual a três anos e situação no contexto da rede SIRGAS-CON (ativa, desativada, descomissionada).

Nesta etapa, as coordenadas das estações de Bom Jesus (BOMJ), Brasília (BRAZ), St. Croix (CRO1), Cuiabá (CUIB), Imperatriz (IMPZ), Kourou (KOUR), La Plata (LPGS), Manaus (NAUS), O' Higgins (OHI2), Presidente Prudente (PPTE), Santiago (SANT), Viçosa (VICO) foram estimadas com injunções relativas na combinação das equações normais pelo módulo ADDNEQ2. As estações da RBMC foram injuncionadas de acordo com a precisão de suas respectivas coordenadas curvilíneas disponibilizadas pelo IBGE, enquanto que as estações SIRGAS-CON foram injuncionadas com a precisão de $1 \mathrm{~mm}$. Em virtude do número de observações superabundantes às precisões obtidas na estimativa dos parâmetros ajustados (coordenadas e velocidades) foram muito otimistas, ou seja, de ordem sub-milimétrica.

Com o intuito de analisar os resultados obtidos, na época 2007,507, realizou-se a comparação com a mais recente solução SIRGAS (SIR09P01), época de referência 2005,0, cujos arquivos de coordenadas e velocidades encontram-se disponíveis no endereço: ftp://ftp.dgfi.badw-muenchen.de/pub/gps/SIRGAS/ e cujas coordenadas foram reduzidas à mesma época da solução estimada. Foram calculadas as discrepâncias entre essas soluções com base nas estações comuns a estas soluções e que apresentaram período superior a três anos de dados.

Verificou-se, que a discrepância entre a solução obtida neste trabalho e a solução SIR09P01, na época 2007,507, é da ordem centimétrica. Observou-se, que embora algumas estações apresentem valores mais discrepantes, a magnitude destas discrepâncias está em torno de $2 \mathrm{~cm}$ para a componente Este, $3 \mathrm{~cm}$ para a componente Norte e de $1 \mathrm{~cm}$ para a componente vertical. As maiores discrepâncias foram observadas na componente Norte para as estações INEG (Águas Calientes, México) e LPAZ (La Paz, Argentina), com 4 e $5 \mathrm{~cm}$ respectivamente; na componente Este para as estações BOGA e BOGT (Bogotá, Colômbia), com discrepâncias em torno de $4 \mathrm{~cm}$ e para a componente vertical, as maiores discrepâncias foram observadas paras as estações BOGA, BOGT, INEG, LPAZ e MERI (Mérida, México), com magnitudes que variam em torno de $5 \mathrm{~cm}$.

Foram geradas as séries temporais das coordenadas das estações envolvidas no processo de integração, com base nas soluções semanais resultantes do processo de combinação das equações normais. Nas Figuras 4 a 6 têm-se as séries temporais para a estação BRAZ, segundo as componentes E, $\mathrm{N}$ e U: 
Figura 4 - Série temporal para a componente Leste da estação BRAZ.

BRAZ

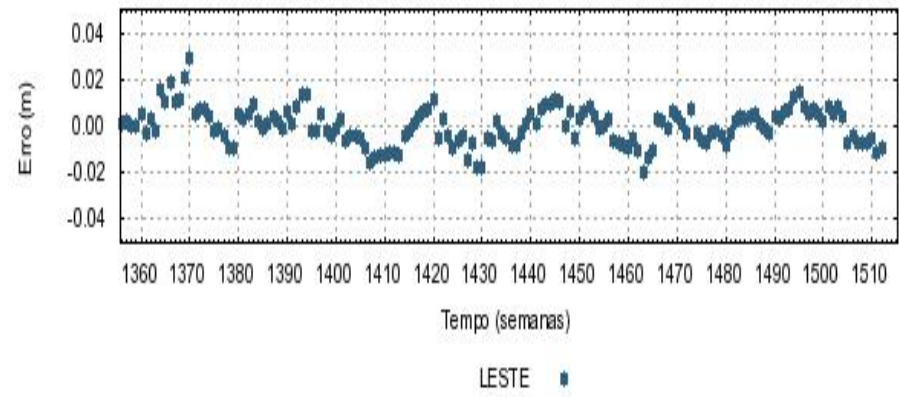

Figura 5 - Série temporal para a componente Norte da estação BRAZ.

BRAZ

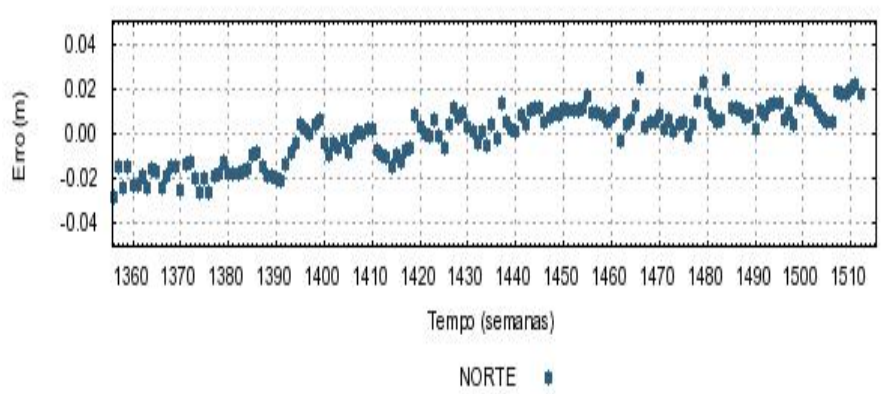

Figura 6 - Série temporal para a componente Vertical da estação BRAZ.

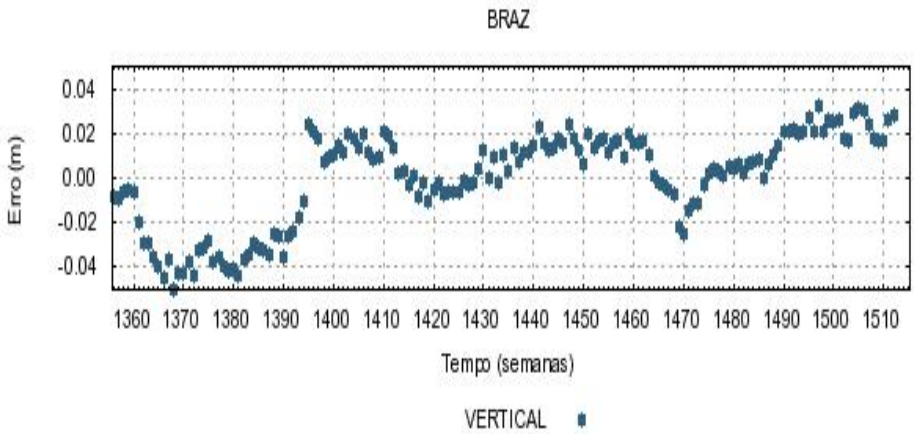

Bol. Ciênc. Geod., sec. Artigos, Curitiba, v. 17, nº 4, p.477-495, out-dez, 2011. 
Verifica-se, que a componente Leste da estação BRAZ (Figura 4) caracteriza uma série sem tendência. Todavia, essa série apresenta, ainda que em menor grau, a sazonalidade, fato que também se evidencia para as componentes Norte (Figura 5) e para a componente Vertical (Figura 6). Além disso, verifica-se que para a componente Norte uma tendência linear no comportamento dos dados.

O comportamento sazonal verificado para a componente altimétrica da estação BRAZ (Figura 6) condiz com os resultados encontrados na literatura, nos quais se verifica que o maior efeito sazonal é obtido para a componente vertical (Bevis et al. (2005); Amiri-Simkooei (2008). Para a estação NAUS foram geradas as séries temporais apresentadas nas Figuras 7 a 9:

Figura 7 - Série temporal para a componente Leste da estação NAUS.

NAUS

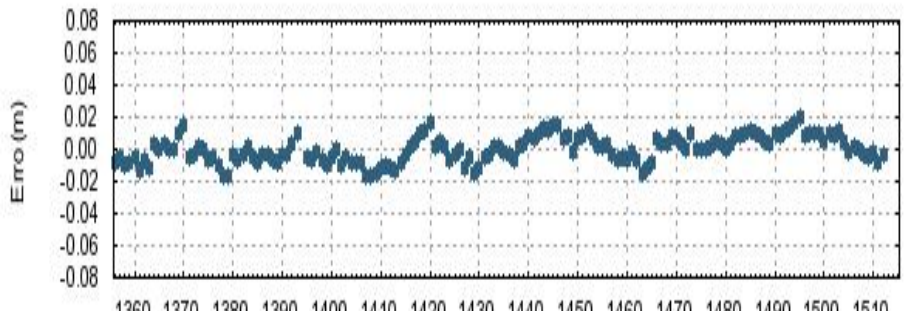

Temp (semanas)

LESTE *

Figura 8 - Série temporal para a componente Norte da estação NAUS.

NAUS

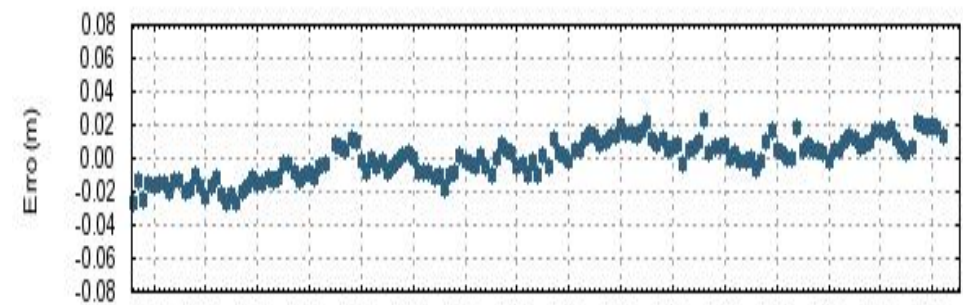

1360137013801390140014101420143014401450146014701480149015001510

Tempo (semanas)

NORTE *

Bol. Ciênc. Geod., sec. Artigos, Curitiba, v. 17, nº 4, p.477-495, out-dez, 2011. 
Figura 9 - Série temporal para a componente Vertical da estação NAUS.

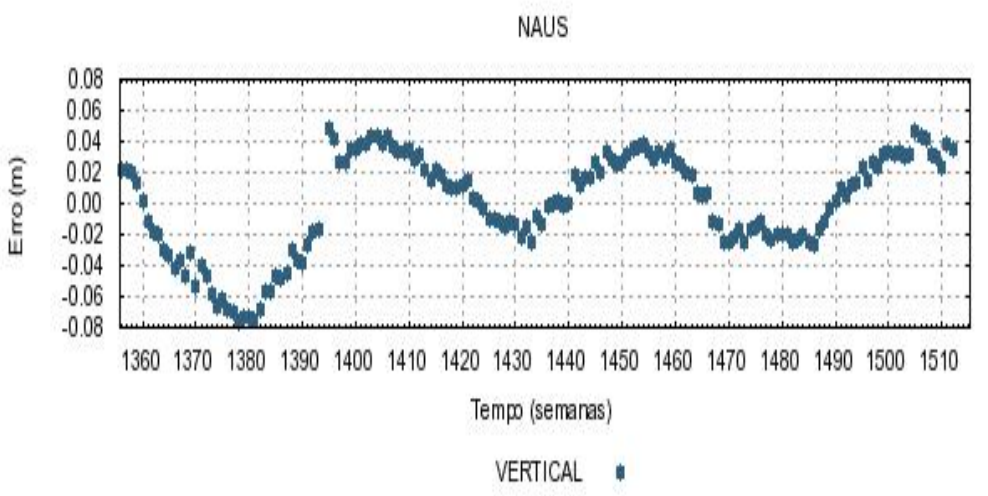

Nas séries temporais da estação NAUS, verifica-se que as componentes Este (Figura 7), Norte (Figura 8) e Vertical (Figura 9) apresentam sazonalidade, sendo que a componente Norte apresenta a tendência. Apesar do período de dados analisados, o comportamento altimétrico da estação NAUS (Figura 9) mostra a relação existente entre a altitude desta estação e dados liminológicos do Rio Amazonas. Nos períodos de cota máxima (maio a junho) o volume de água do Rio Amazonas aumenta, o que conduz a um deslocamento vertical para a componente altimétrica de, aproximadamente, $8 \mathrm{~cm}$. Para os períodos de cota mínima (outubro a janeiro), a componente altimétrica tende a sofrer um deslocamento vertical para cima de no máximo $5 \mathrm{~cm}$, o que caracteriza a anti-correlação existente entre a componente altimétrica da estação com o volume de água do Rio Amazonas. Esta análise é condizente e compatível com resultados encontrados na literatura: Bevis et al. (2005); Costa et al. (2007); Amiri-Simkooei (2008).

Posteriormente, foram estimadas as velocidades de 92 das 193 estações envolvidas ao longo do processo de integração de soluções, uma vez que apresentaram um período e quantidade de dados de no mínimo 3 anos. As maiores discrepâncias são observadas para a componente vertical, podendo atingir até 5 cm/ano como, por exemplo, para a estação UNSA (Salta, Argentina). Como forma de ilustrar o comportamento da velocidade obtida em termos de suas resultantes, no sistema local, em relação à solução SIR09P01 foi gerado um mapa das discrepâncias das velocidades das estações envolvidas, como ilustra a Figura 10:

Em geral, as discrepâncias observadas para as demais estações ficam em torno de $1 \mathrm{a} 2 \mathrm{~cm}$ ao ano, valores que estão de acordo com os encontrados na literatura (FORTES et al., 2006). Além disso, observa-se que o campo de velocidades resultantes gerado pela solução final é muito semelhante ao obtido pela solução SIR09P01, no que concerne ao sentido destes vetores, porém a solução obtida apresenta maior magnitude, fato possivelmente relacionado ao menor período de 
dados que compreende a solução obtida (3 anos de dados) quando comparado a solução SIRGAS

Figura 10 - Mapa das discrepâncias obtidas entre as velocidades das estações estimadas e a solução SIR09P01.

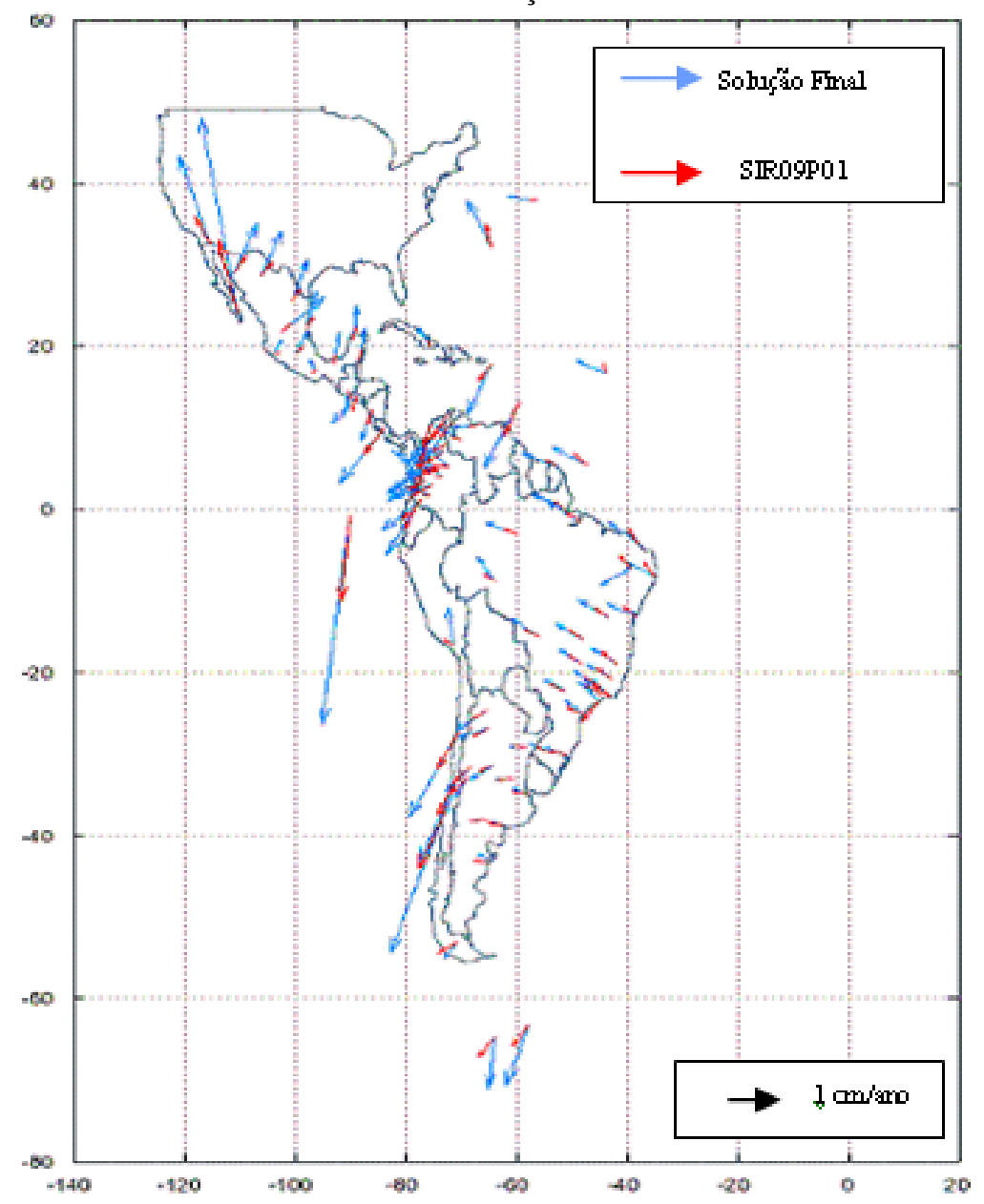

\section{CONSIDERAÇÕES E CONCLUSÕES}

Nesta pesquisa, buscou-se elaborar uma metodologia de integração entre redes SIRGAS (IGS-RNAAC-SIR) GNSS SP, com base no princípio da combinação das equações normais, utilizando-se o software científico Bernese 5.0, e na correção dos 
principais efeitos que afetam o posicionamento GNSS. como, por exemplo, os efeitos de $2^{\mathrm{a}}$ ordem da ionosfera.

No que concerne a aplicação da correção do efeito de $2^{\mathrm{a}}$ ordem, verifica-se que não há uma melhora significativa na obtenção das coordenadas ajustadas, ou seja, as correções atingem valores milimétricos. Contudo, no posicionamento de redes GNSS de alta precisão, tais correções são necessárias, por compreender linhas de bases longas (acima de $100 \mathrm{~km}$ ) e períodos de dados extensos que contemplam períodos de mínima e máxima atividade da ionosfera.

Quanto à obtenção e análise das soluções integradas, verifica-se que embora o período de dados seja de apenas 3 anos (2006 a 2008), pode-se avaliar o comportamento temporal das coordenadas das estações e identificar a presença de efeitos sazonais e locais que afetam, em especial, a componente vertical. Todavia, o estudo da variação temporal das coordenadas requer um período de dados superior ao utilizado nesta pesquisa, bem como à utilização de dados qualitativos associados ao clima, localização geográfica, incidência de fenômenos geofísicos, geomorfologia, em especial em um país com extensões continentais como o Brasil.

No que diz respeito à estimativa da velocidade das estações observou-se que embora os resultados estejam condizentes com a literatura, entende-se que para a obtenção de valores que contribuam a estudos geodinâmicos seja necessário dispor de um período superior de observações, que varia de 5 anos ou mais, como se verifica em trabalhos realizados pelo DGFI e por alguns pesquisadores como Costa (1999).

Finalmente, realizou-se a comparação dos resultados obtidos (coordenadas e velocidades das estações) com a mais recente solução multi-anual SIRGAS, em que se verificou que os valores obtidos são precisos e compatíveis com os resultados determinados por outros centros de processamento.

\section{AGRADECIMENTOS}

Os autores deste trabalho agradecem ao CNPq pelo apoio em forma de bolsa de mestrado junto ao Programa de Pós-Graduação em Ciências Cartográficas da UNESP/FCT e ao IBGE pelas sugestões e críticas a essa pesquisa.

\section{BIBLIOGRAFIA}

AMIRI-SIMKOOEI, A.R. Noise in multivariate GPS position time-series. In: Journal of Geodesy, n. 112, 13p., 2008.

BEVIS, M.; ALSDORF, D.; KENDRICK, E.; FORTES, L. P.; FORSBERG, B.; SMALLEY Jr., R.; BECKER, J. Seasonal fluctuations in the mass of the Amazon River system and Earth's elastic response. In: Journal of Geophysical Research, v. 32, L16308, doi:10.1029/2005GL023491, 2005.

BERNESE GPS SOFTWARE - Version 5.0 (BERNESE). Tutorial. 2007. Disponível em: <http://www.bernese.unibe.ch/download.html>. Acesso em: mar/2008. 
COSTA, S. M. A., Integração da rede geodésica brasileira aos sistemas de referência terrestres. 1999. 170f. Tese de Doutorado em Ciências Geodésicas Universidade Federal do Paraná, Curitiba.

COSTA, S. M. A.; SILVA, A. L.; LAGO, G. N. Primeiro ano de atividades do centro de processamento SIRGAS - IBGE. XXIII Congresso Brasileiro de Cartografia, Rio de Janeiro, Brasil, 21 a 24 de outubro de 2007, p. 173-180.

FORTES, L. P; LAURIA, E.; BRUNINI, C.; AMAYA, W; SÁNCHEZ, L.; DREWES, H.; SEEMÜLLER, W. Current status and future developments of the SIRGAS Project. Geodäsie und Geoinformatik der Universität Hannover, Nr. 258: p. 59-70, 2006. Acesso: mar/2007. Disponível em: $<$ http://www.ife.uniannover.de/mitarbeiter/seeber/seeber_65/pdf_65/fort6.pdf>.

FRITSCHE, M.; DIETRICH, R.; KNÖFEL, C.; RÜLKE, A.; VEY, S.; ROTACHER, M.; STEIGENBERGER, P. Impact of higher-order ionospheric terms of GPS estimates, Geophysical Research Letters, v. 32, L23311, doi: 10.29/2005GL024342, dez. 2005.

GEMAEL, C. Ajustamento de observações: aplicações à Geodésia. Curitiba: UFPR, 1994. 320p.

INSTITUTO BRASILEIRO DE GEOGRAFIA E ESTATÍSTICA (IBGE). Rede Brasileira de Monitoramento Contínuo (RBMC). Disponível em: <http://www.ibge.gov.br/home/geociencias/geodesia/rbmc/RBMC_2009.pdf>. 2009. Acesso: nov/2009.

MARQUES, F. A. Influência da ionosfera no posicionamento GPS: estimativa dos resíduos no contexto de duplas diferenças e eliminação dos efeitos de $2^{\mathrm{a}}$ e $3^{\mathrm{a}}$ ordem da ionosfera. 2008. 150f. Dissertação (Mestrado em Ciências Cartográficas) - Faculdade de Ciências e Tecnologia, Universidade Estadual Paulista, Presidente Prudente.

MONICO, J. F. G. As condições NNT e NNR na realização de um referencial. Curitiba: Boletim de Ciências Geodésicas, v. 11, nº 1, p. 45-52, 2005.

MONICO, J. F. G. Fundamentos matemáticos envolvidos na realização do ITRS. Curitiba: Boletim de Ciências Geodésicas, v. 12, nº 2, p. 337-351, 2006.

SISTEMA DE REFERÊNCIA GEOCÊNTRICO PARA AS AMÉRICAS (SIRGAS). 2009. Disponível em: <http://www.sirgas.org/>. Acesso: mar/2009.

STEINGENBERGER, P.; ROTHACHER, M.; DIETRICH, R.; FRITSCHE, M.; RÜLKE, A.; VEY. S. Reprocessing of a global GPS network. Journal of Geophysical Research, vol. 111. 2006.

(Recebido em julho de 2011. Aceito em outubro de 2011.) 